\title{
Immunoglobulin use in immune deficiency in the UK: a report of the UKPID and National Immunoglobulin Databases
}

\author{
Authors: Ben Shillitoe, ${ }^{\mathrm{A}, \mathrm{B}, *}$ Rob Hollingsworth, ${ }^{\mathrm{C}}$ Mark Foster, ${ }^{\mathrm{D}}$ Tomaz Garcez, ${ }^{\mathrm{E}, *}$ David Guzman, ${ }^{\mathrm{F}, *}$ ] David Edgar, \\ and Matthew Buckland ${ }^{\mathrm{H}, *}$
}

Supply of immunoglobulin in the UK faces pressures due to increasing demand, cost and variable supply. This paper describes immunoglobulin replacement therapy (IGRT) in primary immunodeficiency (PID) and secondary immunodeficiency (SID) to assist in the ongoing planning of UK immunoglobulin provision. A retrospective analysis of the National Immunoglobulin Database and the UKPID registry was carried out. In total, 3,222 patients are registered as receiving IGRT for immunodeficiencies. Predominately antibody disorders made up the largest diagnostic category (61\% of patients). The total cost of IGRT for immunodeficiency for 2015/16 was $£ 40,673,350$; an average annual cost of $£ 1,099,254$ per centre and $€ 12,124$ per PID patient. SCIg accounted for $43.8 \%$ and $50.1 \%$ of IGRT, with home therapy accounting for $42.7 \%$ and $57.5 \%$ of place of therapy in the National Immunoglobulin Database and UKPID registry respectively. In 2015/16 use of immunoglobulin in SID increased by $24 \%$ over the previous financial year. The overall trends of increasing demand in immunology are mirrored in other specialties, most notably neurology and haematology. These data are the first national overview of IGRT for immunodeficiencies, providing a valuable resource for clinicians and policy makers in the ongoing management of UK immunoglobulin supply.

KEYWORDS: Immunoglobulin, immunodeficiency, intravenous, subcutaneous, England

Authors: ${ }^{\text {A }}$ linical research associate, Institute of Cellular Medicine, Newcastle University, Newcastle upon Tyne, UK; ${ }^{B}$ clinical research associate, Great North Children's Hospital, Newcastle upon Tyne Hospitals Trust, Newcastle upon Tyne, UK; C managing director, Medical Data Solutions and Services, UK; ${ }^{\text {Dhealthcare }}$ informatics analyst, Medical Data Solutions and Services, UK; ${ }^{E}$ consultant immunologist, Manchester University NHS Foundation Trust, Manchester, UK; F software engineer, UCL Centre for Immunodeficiency, Royal Free Hospital, London, UK; ${ }^{G}$ consultant immunologist, Regional Immunology Service, The Royal Hospitals, Belfast Health and Social Care Trust and Queen's University Belfast, Belfast, Northern Ireland, UK; ${ }^{H}$ consultant immunologist, Institute of Immunity and Transplantation, UCL, London, UK; "on behalf of the UKPID Registry Committee, UKPIN

\section{Introduction}

In 1952, Colonel Ogden Bruton described the first case of X-Linked agammaglobulinaemia (XLA) and treated this young boy with immunoglobulin replacement therapy (IGRT), which had only recently been made available. ${ }^{1}$ This case report is often cited as the birth of clinical immunology and since that time immunoglobulin replacement therapy (IGRT) has become the mainstay of treatment for numerous diseases across the primary immunodeficiency (PID) spectrum. ${ }^{1,2}$ Over 300 PIDs are now recognised, with approximately $60 \%$ associated with hypogammaglobulinaemia. ${ }^{3}$ Aside from its use in PID, immunoglobulin therapy also plays a major role in the treatment of secondary immunodeficiencies (SID). This can be either following treatment for connective tissue disease (especially with rituximab) or as a manifestation of, or as a consequence of treatment for, lymphoreticular malignancy and other haematological diseases. ${ }^{4}$ In other medical disciplines, most notably neurology, a significant amount of immunomodulatory, rather than replacement, immunoglobulin is prescribed in the UK.

Since its first description in 1944, production of immunoglobulin has undergone many changes and improvements over the decades. ${ }^{5}$ While Bruton used subcutaneous IGRT, from 1950 to 1980 IGRT was predominantly administered as an intramuscular (IM) preparation. Changes in the production process allowed IGRT to be given via the intravenous (IV) route in the early 1980s, with the UK switching over to IV preparations in 1982. ${ }^{6,7}$ IV preparations are less painful, allowing larger volumes to be administered and therefore resulting in higher trough IgG levels. These higher IgG trough levels dramatically reduced invasive infection rates, thereby improving survival outcomes. ${ }^{8,9}$ Sadly, this period coincided with the emergence of HIV and, in addition to hepatitis $C$, contaminated products placed a significant burden on cohorts dependent on donor blood products. While there are no confirmed reports of immunoglobulin-transmitted HIV, contracting hepatitis $C$ through contaminated immunoglobulin has been confirmed. These iatrogenic infections led to multiple international inquiries and millions of pounds set aside or awarded for compensation. ${ }^{10}$ The safety profile of donor immunoglobulin products has since significantly improved with screening of donors and through improvements in the production process with exposure to low $\mathrm{pH}$, pasteurisation, detergents and viral filtration. ${ }^{11}$ With improved production protocols, these risks of 
contamination with blood-borne viruses have dramatically fallen, although a small theoretical risk still exists, particularly of prionborne disease. ${ }^{12,13}$ The risk of variant Creutzfeldt-Jakob disease ( $\mathrm{VCJD}$ ) in the UK has led to the decision that no IGRT is sourced from UK donor pools. To date, no PID patient within the UK has demonstrated clinical evidence of $\mathrm{VCJD}$ or abnormal prion protein in tested tissues. ${ }^{14}$

Once the decision has been made to commence IGRT in a patient, the need for continued treatment is kept under review; however, it is likely that most will remain on IGRT for the remainder of their lives, whether for primary or secondary immunodeficiency. Patients' clinical outcomes and quality of life are often dependant, sometimes entirely, on their IGRT. The demands made upon the national immunoglobulin supply from treating immune deficiencies could have significant knock-on effects for other specialties for whom a consistent supply of immunoglobulin is also needed. With significant increases in the use of replacement immunoglobulin there have been concerns at being able to meet the demands for immunomodulatory treatment, such as ITP (immune thrombocytopenia), CIDP (chronic inflammatory demyelinating polyneuropathy), MMN (multifocal motor neuropathy). While there may be alternative modalities of treatment for patients undergoing immunomodulation with IVIg, the tolerability, efficacy and therapeutic index of the alternatives do not make them a preferred choice. Therefore, the NHS needs to ensure a secure and readily available supply of immunoglobulin products to ensure optimal patient outcomes across all licensed indications.

These demands place considerable pressure on the procurement and supply of immunoglobulin within the UK. In the early 2000s significant concerns were raised regarding variable supply, increasing demand and high costs of immunoglobulin therapy. In response, the Department of Health produced a review in 2006 which led to the creation of two complementary programmes; one based on securing supply and the second, the Demand Management Programme for Immunoglobulin, overseeing the meeting of demand within in the UK. ${ }^{15}$ Setting up the Demand Management Programme necessitated the creation of the National Immunoglobulin Database. In a separate initiative, The United Kingdom Primary Immunodeficiency Network (UKPIN) established an online patient registry (UKPID Registry) in 2008.

This study analyses these two national UK databases and aims to describe current practices regarding immunoglobulin therapy in the UK within clinical immunology.

\section{Methods}

This study presents data from two UK databases, the UK Primary Immunodeficiency (UKPID) registry and the National Immunoglobulin Database.

\section{UKPID registry}

The UKPID registry collects information from 37 recognised UK immunology centres (covering $97 \%$ of UK PID centres). ${ }^{16}$ It is the primary registry for PID patients in the UK and offers researchers a single repository of data on UK PID patients. ${ }^{16}$ It is also the only national registry to collect clinical data on patients with secondary antibody deficiency. It is funded and overseen by the UK Primary Immunodeficiency network (UKPIN) (www.ukpin.org.uk/registry/ registry-intro). Anonymised data are collected on individual patients in relation to diagnosis, treatment, investigations, infections and complications. Data entry is cumulative and entries are updated on an annual basis. The development, ongoing management and technical database structure of the registry has been described previously. ${ }^{16-19}$ Multicentre research ethics (MREC) approval was obtained in 2004 for the ESID (European Society for Immunodeficiencies) online database (MREC number: 04/MRE07/68). It was subsequently felt that the creation of a standalone UK registry would allow the addition of variables that are of importance and interest to UK clinicians and researchers that may not otherwise be available from the ESID registry, while data common to both is shared under appropriate data protection arrangements. Approvals have been amended to reflect the establishment of a UK based database. ${ }^{16}$ Data presented is cumulative up until April 2017.

\section{National Immunoglobulin Database}

As part of the newly created Demand Management Programme, the National Immunoglobulin Database was launched in June 2008 (http://igd.mdsas.com). The technical set-up of the database has been described previously. ${ }^{20}$ It is managed by Medical Data Solutions and Services (MDSAS, www.mdsas.com). This database produces a yearly report on overall immunoglobulin use in England and is used extensively by NHS England to support commissioning and therapy initiatives for immunoglobulin. This database is completed prospectively for all immunoglobulin product administration in England. It should therefore provide near complete information on intravenous (IVIg) and subcutaneous (SCIg) usage in England. For the purposes of this study, records were filtered to include immunology use only. Data for the National Immunoglobulin Database was analysed for the time period April 2013 to April 2016.

\section{Analysis}

These two dataset sets were compared to provide enhanced information on the current distribution and management of immune deficiency patients in the UK, with both datasets contributing data for patients receiving their therapy in England, and the UKPID registry providing data for patients who received their therapy in England, Wales, Scotland and Northern Ireland. The UKPID registry provided detailed diagnostics and outcome data, with the National Immunoglobulin Database providing reliable figures on IGRT usage, dosing, as well as longitudinal trends.

Variables and results are compared for the two databases. Where only one database source was used, this is clearly stated within the text. Data was also produced for specific centres to provide external comparators and identification of any significant variation in practice. Each centre is anonymised throughout this report, identified by unique ID numbering 1 through to 37 .

IGRT usage was broken down by diagnosis, contributing centre, sex and age. Comparisons were made among IVIg versus SCIg. Doses and levels are displayed as median and interquartile range (IQR). Where data were only available for a subset of the patients the denominator is stated clearly within the text.

Data were analysed using Microsoft Excel 2016, GraphPad Prism v6.01 (www.graphpad.com) and Stata v15.1 (www.stata.com).

No additional ethical approval was necessary for this study. The ethical approval / consent process for the UKPID registry allows the use of the registry data to be used for research / published without any further approvals aside from approvals from the registry steering committee which were obtained. 


\section{Results}

A total of 2,711 patients are recorded as receiving IGRT on the UKPID registry compared to 3,222 on the National Immunoglobulin Database. A breakdown of the patients in the UKPID registry by IUIS category is shown in Supplementary file 1. Predominately antibody disorders account for $60.9 \%$ of patients within the UKPID registry. Demographics of each database by age group and sex are similar (Supplementary file 2). There is an approximate $50: 50$ split between male and female in both registries. The proportion of under- $18 \mathrm{~s}$ is $11.4 \%$ and $14.5 \%$ in the UKPID and National Immunoglobulin Database respectively.

There is a marked variation in the number of patients receiving IGRT among immunology centres (Supplementary file 3), most likely as a result of differing catchment populations. The median number of patients receiving IGRT at each centre was 50 (IQR 28-111) for the UKPID registry and 93 (IQR 46-152) for the National Immunoglobulin Database.

The most common diagnosis in each dataset was common variable immunodeficiency (CVID) accounting for $50 \%$ of patients in the UKPID registry and $37.2 \%$ of patients in the national immunoglobulin database. Although predominately a PID database, the UKPID registry does collect information on secondary immunodeficiencies and for both databases, these cases were the second most common diagnosis for patients receiving IGRT (12\% UKPID, 16.1\% National Immunoglobulin Database). Breakdown by diagnosis per the two databases is shown in Table 1. Within the National Immunoglobulin Database $40.5 \%$ of patients are cited as 'Unclassified hypogammaglobulinaemia'. This is due to restrictions in diagnostic classification within this database.

Table 1. Breakdown of diagnosis for those receiving immunoglobulin replacement therapy according to each database

$\begin{array}{lll}\text { Diagnosis } & \begin{array}{l}\text { UKPID } \\ \text { Registry }\end{array} & \begin{array}{l}\text { National } \\ \text { Immunoglobulin } \\ \text { Database }\end{array} \\ \text { CVID } & 1,358(50.1 \%) & 1,199(37.2 \%) \\ \begin{array}{l}\text { Secondary } \\ \text { immunodeficiency }\end{array} & 326(12.0 \%) & 519(16.1 \%) \\ \text { XLA } & 198(7.3 \%) & \text { N/A } \\ \text { SPAD } & 121(4.5 \%) & 132(4.1 \%) \\ \text { Unclassified } & 197(7.3 \%) & 1,306(40.5 \%) \\ \text { hypogammaglobulinaemia } & & 17(0.5 \%) \\ \text { Thymoma with ID } & 68(2.5 \%) & \text { N/A } \\ \text { IgG subclass } & 44(1.6 \%) & 36(1.1 \%) \\ \text { HIGM } & 41(1.5 \%) & 13(0.4 \%) \\ \text { Post HSCT } & 0(0 \%) & \text { N/A } \\ \text { IgA deficiency } & 13(0.5 \%) & \text { N/A } \\ \text { IgA and IgG subclass } & 10(0.4 \%) & 3,222 \\ \text { deficiency } & & \mathbf{2 , 7 1 1}\end{array}$

CVID = Common variable immunodeficiency; $\mathrm{HIGM}=$ hyper IgM; $\mathrm{HSCT}=$ haematopoietic stem cell transplantation; ID = immunodeficiency; SPAD = specific IgG deficiency; XLA = X-Linked agammaglobulinaemia
The UKPID database shows a median Ig dose of $579.4 \mathrm{mg} /$ $\mathrm{kg} / \mathrm{month}$ (IQR 457.1-724.1) at a median interval of 3 weeks (IQR 1-3) (Fig 1). Median IVIg trough level was 986 mg/dL (IQR 800-1180). Median random SCIg levels were $900 \mathrm{mg} / \mathrm{dL}$ (IQR 640-1120).

Using the National Immunoglobulin Database data, the total usage of immunoglobulin for PID in 2015/16 was 1,196,275 g, representing $26 \%$ of total immunoglobulin use within England. Based on a calculated average cost per gram of $£ 34$, this equates to annual cost of $£ 40,673,350$. This equates to an average annual cost of $£ 1,099,254(32,331 \mathrm{~g})$ per centre and $£ 12,614$ (371 g) per PID patient. Secondary antibody deficiencies accounted for $6 \%$ $(285,392 \mathrm{~g})$ of the total immunoglobulin used in 2015/16 making it the fourth commonest diagnosis in the national immunoglobulin database, by volume of immunoglobulin used (the second and third commonest diagnoses being CIDP and MMN respectively). This represents a $24 \%$ increase from the previous financial year, 2014/15

There is marked variation in the ratio of subcutaneous to intravenous therapy among centres, mirrored by their variation in preferred place of therapy (home versus hospital) shown in Fig 2. Per centre, the median ratio of subcutaneous (SC) to IV therapy was 1.13 (IQR 0.66-1.90) and the median ratio of home to hospital therapy was 1.72 (IQR 1.11-3.27). Overall, $43.8 \%$ and $50.1 \%$ of patients receive their IGRT by the subcutaneous route in the National Immunoglobulin Database and UKPID registry respectively. $42.7 \%$ and $57.5 \%$ of patients receive their therapy at home in the national immunoglobulin and UKPID database respectively (Fig 3).

Both databases recorded the most common IVIg preparation as Privigen (26.4\% UKPID, 36.3\% National Immunoglobulin Database). They also both record Hizentra as the most common SCIg prescribed (41.7\% UKPID, 48.3\% National Immunoglobulin Database).

\section{Discussion}

Within the National Immunoglobulin Database, clinical immunology accounts for $35 \%$ of all immunoglobulin use, second only to neurology (42\%). Primary immunodeficiency, as a group of disorders, accounts for $26 \%$ of the 4.6 million grams of immunoglobulin used in 2015-16. ${ }^{21}$ The remaining immunoglobulin therapy used by clinical immunology will be largely related to IGRT in secondary immunodeficiency and as immunomodulation in a range of autoimmune and inflammatory disorders. In the 2015-16 financial year, the total cost of immunoglobulin use in England was $£ 165$ million. It is predicted that by the financial year 2017-18, the total immunoglobulin cost per annum will be £190-200 million. ${ }^{21}$ There has been a consistent rise in Ig sales of between $5-10 \%$ since the National Immunoglobulin Database was created. This is in conjunction with a $6.8 \%$ reduction in the average price paid per gram between 2011 and 2016 as a result of lower contract prices and product switching. ${ }^{21}$ The current national immunoglobulin framework contract supplies 17 different products through six contracted suppliers. ${ }^{21}$

In June 2017, NHS England made significant changes to immunoglobulin procurement and, in combination with product changes from manufacturers, a significant number of UK patients will have to undergo a change of immunoglobulin product over the coming 12 months. Some changes are as a result of product 


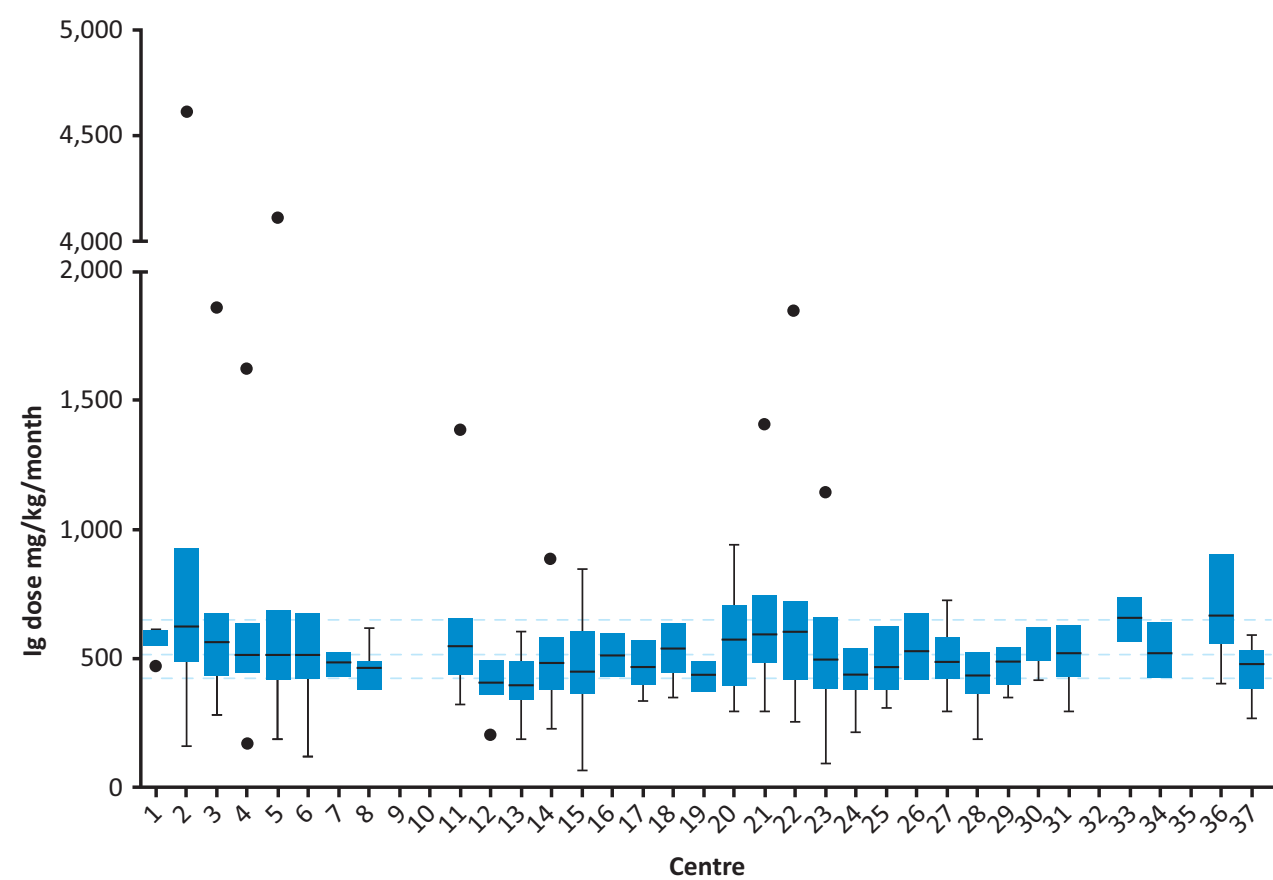

Fig 1. Turkey box plot of Ig dose per centre $(\mathrm{mg} / \mathrm{kg} /$ month). Each box represents median and IQR with range (whiskers) and outliers (dots). Top, middle and bottom horizontal dashed lines represent group 75th centile, median and 25 th centile respectively. changes from manufacturers (eg Shire's Subcuvia 16\% will be replaced with Cuvitru 20\%), or some products being withdrawn from the market (eg BPL Ltd's Vigam 5\%). In addition, changes to the national framework agreements have resulted in changes to which products NHS England will procure. Although the two databases have differences in the way they record the brand of immunoglobulin, both agree that Privigen and Hizentra are currently the most common IV and SC preparations prescribed for IGRT in clinical immunology.

UK immunoglobulin has been sourced from plasma donated outside the UK since the bovine spongiform encephalopathy (BSE) outbreak due to the potential risk of $\mathrm{V} C \mathrm{JD}$ transmission. Plasma used to fractionate immunoglobulin is sourced from countries that have not had BSE outbreaks and have only background (sporadic) CJD case numbers. The UK is therefore reliant on a global market for the supply of plasma products. The recent shortage of immunoglobulin has been in part due to plasma and plasma products being diverted to more lucrative markets at the same time that limited numbers of plasma donation centres have been opened.

Modern IGRT is available either through the intravenous route (usually on a 3-4 weekly basis) or the SC route (usually on a weekly basis by pump, but increasingly by rapid push more frequently). Recently, the technique of preinfusion of SCIg with recombinant human hyaluronidase (facilitated SCIG, fSCIg) may also offer the infusion of SCIG to be extended to 2-4 weeks, similar to IVIg, although fSCIg is not currently commissioned in England. ${ }^{22}$ With adequate training and support either route can be administered at home or hospital. There are various advantages and disadvantages to either route or site of IGRT. The decision to choose one over the other will be individual to each patient, depending on a range of factors including clinical indication, venous access, compliance, patient preference and access to secondary care. It is likely that patients' circumstances will change over the course of their IGRT therapy and site and/ or route of IGRT similarly may have to be altered. These results show an almost equal split between IVIg and SCIg among the two databases, although home therapy was often preferred in these data with a median ratio of hospital to home therapy of 1.72 per centre. In total, $42.7 \%$ and $57.5 \%$ of patients received their therapy at home in the National Immunoglobulin and UKPID databases respectively. It is worth noting that the recent shortage of immunoglobulin in the UK has exclusively been related to IVIg supply. A progressive switch of patients to SC immunoglobulin, both for replacement and immunomodulation, has the potential to reduce pressure on the demand for IVIg in the UK and has additional advantages in reducing hospital associated costs with IVIg administration to the NHS. ${ }^{23}$ The IGRT dosing for all centres appear to fall largely within expected boundaries except for a small number of outliers, possibly due to miscoding of diagnosis and/or speciality. The corresponding median trough level from the UKPID database of $986 \mathrm{mg} /$ $\mathrm{dL}$ and random IgG trough levels from the SCIg patients of $900 \mathrm{mg} / \mathrm{dL}$ appear satisfactory and in line with the published literature describing adequate dosing in primary antibody deficiencies. ${ }^{24}$ It should be noted that modern practice would dictate individualising IGRT in patients to adequately prevent infections for that individual rather than a blanket target IgG trough level. ${ }^{25,26}$

Comparing Table 1 and the table in Supplementary file 1, there are 52 patients within the UKPID registry who have a predominately antibody disorder but are not receving IGRT. There are patients with antibody deficiency who decline IGRT as part of patient choice and are managed with antimicrobial therapy as required or prophylactically.

There are some limitations in this report with some mismatching between the databases as described in this manuscript. This is due to a variety of reasons: differences in the collection time window, 


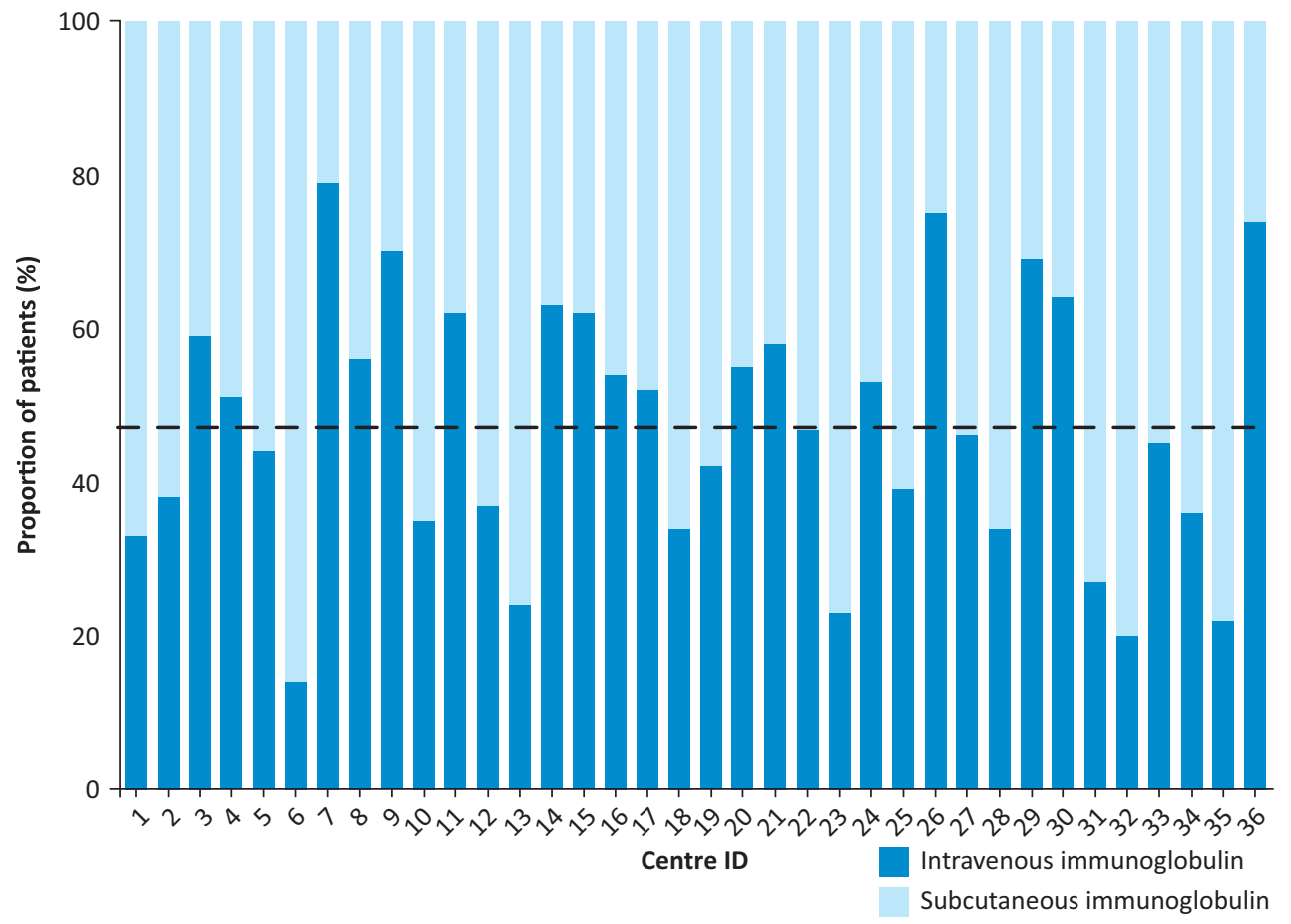

Fig 2. Ratio of route and place of IGRT. Median percentage for IVIg and hospital therapy usage demonstrated by the dashed, blackline. Centre numbers shown along the horizontal axis.

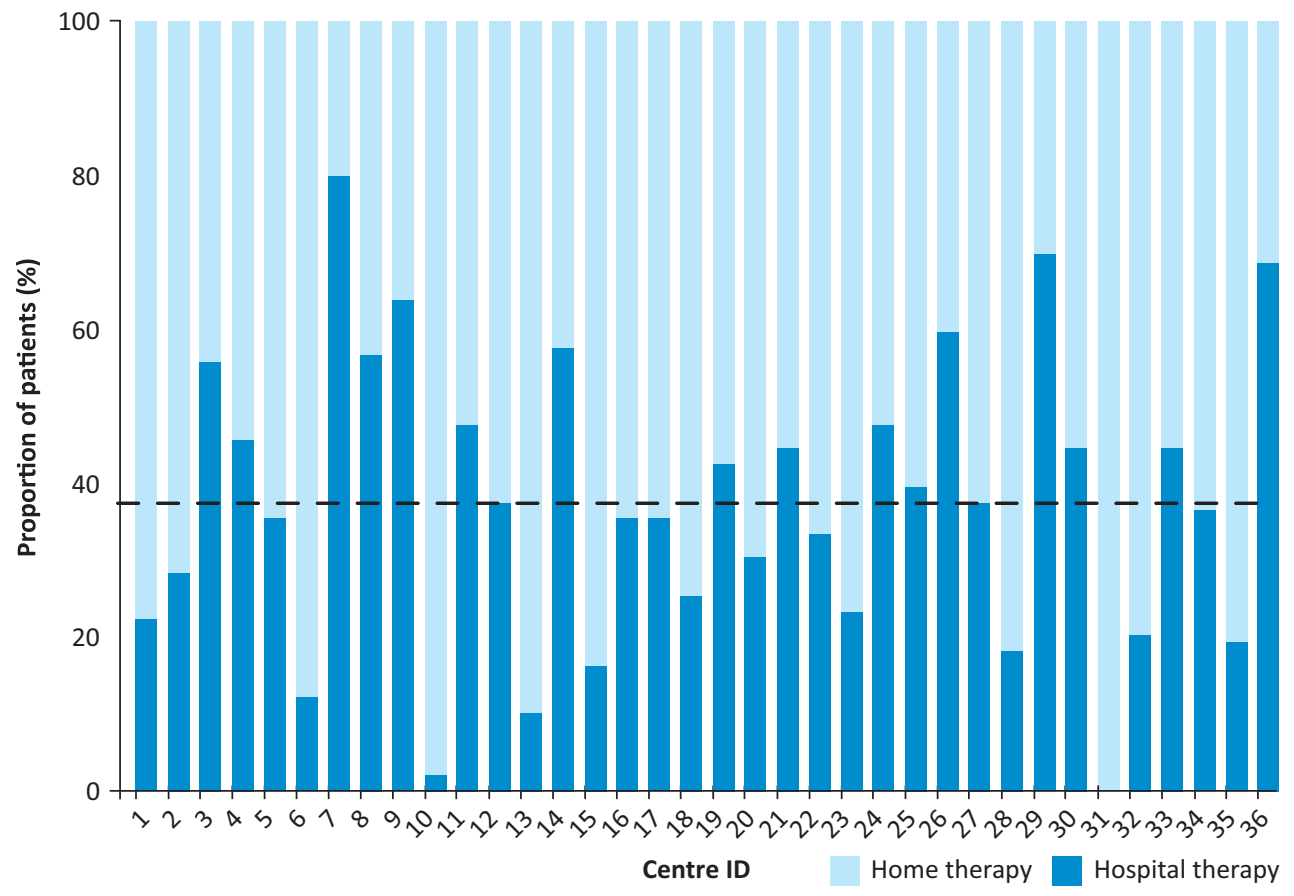

variable data entry between database fields, Scotland and Wales not contributing to the National Immunoglobulin Database and differences in grouping some centres together within single trusts. The National Immunoglobulin Database is considered an accurate overview of immunoglobulin use, and is estimated to capture approximately $90 \%$ of national immunoglobulin use when compared to data recorded with the Commercial Medicines Unit (CMU). ${ }^{21}$ In general, the diagnostic data in the UKPID is of greater quality and accuracy than the National Immunoglobulin Database. Patients with secondary immunodeficiencies contribute a significant proportion of the patients described here $(12.0 \%$ and $16.1 \%$ in the UKPID and National immunoglobulin databases respectively). Their data has been included to demonstrate the significant workload this group contribute to UK clinical immunology and their subsequent demand on the national immunoglobulin supply.

This report is the first comparison and combined analysis of the two major data sources detailing IGRT for immunology within the UK. Allowing for the limitations described above, the data validation between the two datasets shown here demonstrates their high level of data coverage and detail. These data make a vital contribution to discussions and planning for 
UKPID
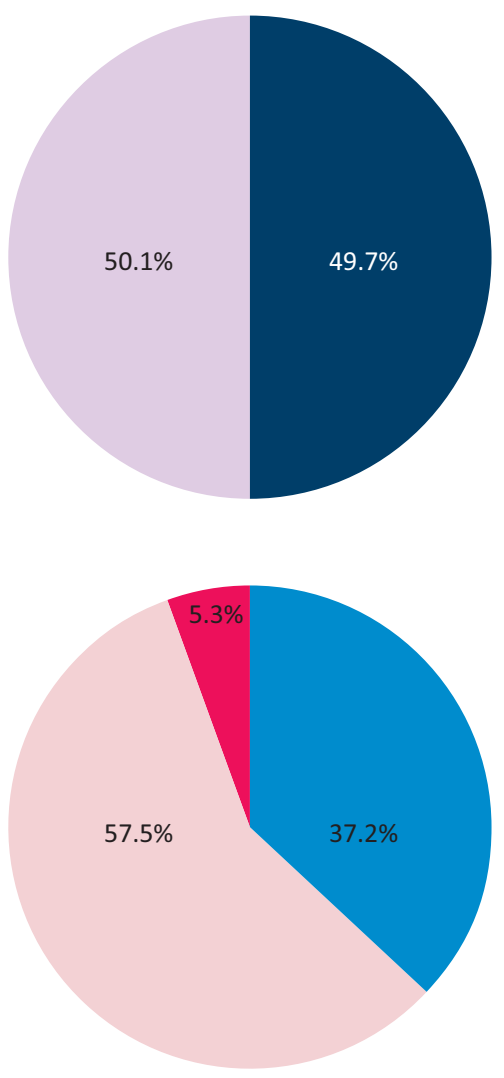

National Immunoglobulin Database
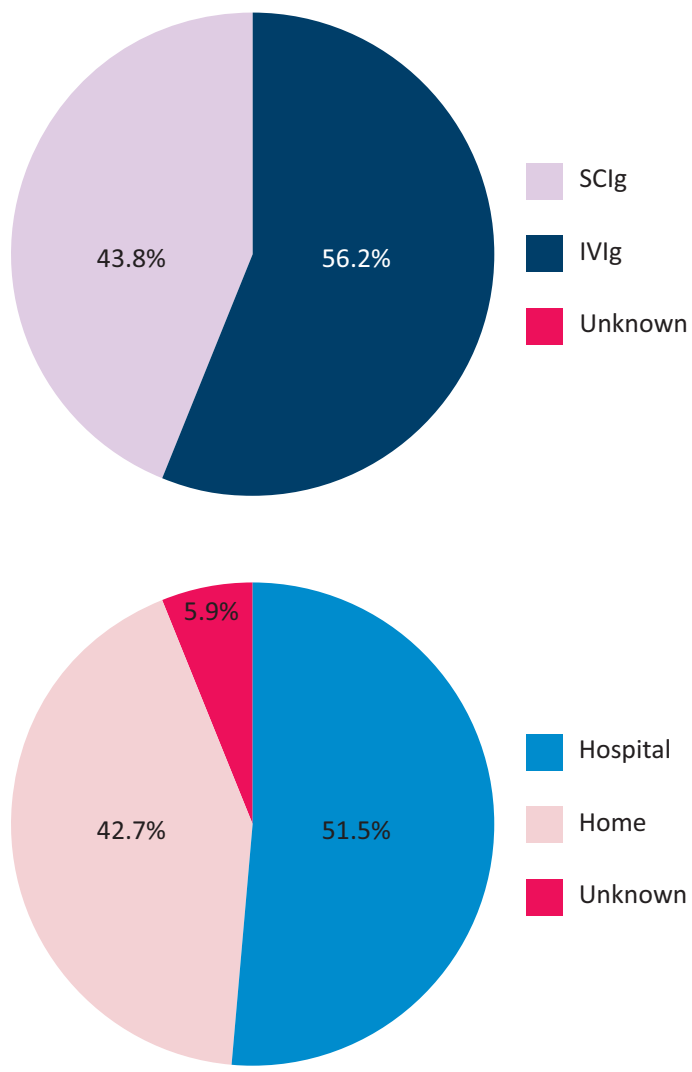

Fig 3. Breakdown of site and location of IGRT by database. future immunoglobulin use within the UK. These data provide an accurate overview detailing immunoglobulin use in the UK for clinical immunology both at national and individual centre level. While the National Immunoglobulin Database provides good quality data on the amount of immunoglobulin used, it is only with the clinical data provided by the UKPID registry that clinicians and policy makers can make an accurate review of current practices and make robust plans for future UK immunoglobulin supply. Cost and supply will continue to put pressure on the UK immunoglobulin provision, as well as any future changes in product availability or changes to suppliers. These data, and the demands made by clinical immunology upon the immunoglobulin supply, also have significant implications for other specialties for whom immunoglobulin therapy plays a major role, most notably neurology and haematology. Within these other specialties, immunoglobulin is often used in an immunomodulatory role which often requires much higher doses than in IGRT, placing further strain on UK immunoglobulin supply. The impact of Brexit may also put additional pressures on supply and careful attention to IGRT supply must be made during the process of exiting Europe. These data here, along with the ongoing data collection by the two databases provides valuable information for clinicians and policy makers in helping to ensure reliable and adequate supply of immunoglobulin products for PID patients within the UK.

\section{Supplementary material}

Additional supplementary material may be found in the online version of this article at www.clinmed.rcpjournal.org:
S1 - Current breakdown of patients in the United Kingdom Primary Immunodeficiency Registry (UKPID) by International Union of Immunological Societies (IUIS) category.

S2 - Demographics of each database by sex and age groups.

S3 - Comparison of IGRT numbers for UKPID and National Immunoglobulin Database by centre.

\section{References}

1 Bruton OC. Agammaglobulinemia. Pediatrics 1952;9:722-8.

2 Chapel H, Prevot J, Gaspar HB et al. Primary immune deficiencies principles of care. Front Immunol 2014;5:1-12.

3 Picard C, Al-Herz W, Bousfiha A et al. Primary immunodeficiency diseases: an update on the classification from the International Union of Immunological Societies Expert Committee for Primary Immunodeficiency 2015. J Clin Immunol 2015;35:696-726.

4 Makatsori M, Kiani-Alikhan S, Manson AL et al. Hypogammaglobulinaemia after rituximab treatment - incidence and outcomes. QJM 2014;107:821-8.

5 Cohn EJ, Oncley JL, Strong LE, Hughes WL, Armstrong SH. Chemical, clinical, and immunological studies on the products of human plasma fractionation. I. The characterization of the protein fractions of human plasma. J Clin Invest 1944;23:417-32.

6 Cunningham-Rundles C. Immunoglobulin Replacement Therapy. In: Webster ADB (eds), Immunodeficiency and Disease. Dordrecht: Springer Netherlands, 1988:43-60.

7 Hermaszewski R, Webster A. Primary hypogammaglobulinaemia: a survey of clinical manifestations and complications. Q J Med 1993;86:31-42.

8 Pirofsky B, Campbell SM, Montanaro A. Individual patient variations in the kinetics of intravenous immune globulin administration. J Clin Immunol 1982;2(Suppl 2):7S-14S. 
9 Garbett ND, Currie DC, Cole PJ. Comparison of the clinical efficacy and safety of an intramuscular and an intravenous immunoglobulin preparation for replacement therapy in idiopathic adult onset panhypogammaglobulinaemia. Clin Exp Immunol 1989;76:1-7.

10 Pappenheim K. UK inquiry should establish why contaminated blood products were given to people with haemophilia. BMJ 1999;319:52.

11 Sriaroon P, Ballow M. Immunoglobulin replacement therapy for primary immunodeficiency. Immunol Allergy Clin North Am 2015;35:713-30.

12 Healey $\mathrm{C}$, Chapel $\mathrm{H}$. Intravenous immunoglobulin and hepatitis $\mathrm{C}$ virus: the British episode. Clin Ther 2016;18:93-5.

13 Biesert L. Virus validation studies of immunoglobulin preparations. Clin Exp Rheumatol 1996;14(Suppl 1):S47-52.

14 The National CJD Research \& Surveillance Unit. Creutzfeldt-Jakob disease surveillance in the UK - 25th Annual Report. NCJDRSU, 2016.

15 Fitzharris P, Hurst M. Commentary: Controversies in the Department of Health's clinical guidelines for immunoglobulin use. BM] 2008;337:a1851.

16 Edgar JDM, Buckland M, Guzman D et al. The United Kingdom Primary Immune Deficiency (UKPID) Registry: report of the first 4 years' activity 2008-2012. Clin Exp Immunol 2014;175:68-78.

17 Eades-Perner A-M, Gathmann B, Knerr V et al. The European internet-based patient and research database for primary immunodeficiencies: results 2004-06. Clin Exp Immunol 2007;147:306-12.

18 Gathmann B, Grimbacher B, Beaute ] et al. The European internet-based patient and research database for primary immunodeficiencies: results 2006-2008. Clin Exp Immunol 2009;157(Suppl 1):3-11.

19 Guzman D, Veit D, Knerr V et al. The ESID Online Database network. Bioinformatics 2007;23:654-5.
20 Department of Health. First National Immunoglobulin Database Report (2008-2009) http://igd.mdsas.com/wp-content/uploads/ DH_ImmunoDatabaseReport-2010.pdf [Accessed 14 ]anuary 2018].

21 Medical Data Solutions and Services. Immunoglobulin Database Report 2015/2016. MDSAS, 2017

22 Jolles $\mathrm{S}$. Hyaluronidase facilitated subcutaneous immunoglobulin in primary immunodeficiency. Immunotargets Ther 2013;2:125-33.

23 van Schaik IN, Bril V, van Geloven N et al. Subcutaneous immunoglobulin for maintenance treatment in chronic inflammatory demyelinating polyneuropathy (PATH): a randomised, double-blind, placebo-controlled, phase 3 trial. Lancet Neurol 2018;17:35-46.

24 Orange JS, Grossman W], Navickis RJ, Wilkes MM. Impact of trough IgG on pneumonia incidence in primary immunodeficiency: A meta-analysis of clinical studies. Clin Immunol 2010;137:21-30.

25 Lucas M, Lee M, Lortan ] et al. Infection outcomes in patients with common variable immunodeficiency disorders: relationship to immunoglobulin therapy over 22 years. J Allergy Clin Immunol 2010;125:1354-60.e4.

26 Chen Y, Stirling RG, Paul E et al. Longitudinal decline in lung function in patients with primary immunoglobulin deficiencies. J Allergy Clin Immunol 2011;127:1414-7.

Address for correspondence: Dr Ben Shillitoe, Newcastle University Institute of Cellular Medicine, c/o Roz Scott, Paediatric Immunology, Floor 4, Block 2 Clinical Resource Building, Royal Victoria Infirmary, Newcastle upon Tyne NE1 4LP, UK.

Email: b.shillitoe2@ncl.ac.uk 